\title{
Effect of the COVID-19 Pandemic Preparation and Response on Essential Health Services in Primary and Tertiary Healthcare Settings of Amhara Region, Ethiopia
}

\author{
Wendemagegn Enbiale, ${ }^{1,2}$ Seid Getahun Abdela, ${ }^{3 *}$ Meaza Seyum, ${ }^{4}$ Dereje Bedanie Hundie, ${ }^{1}$ Kassawmar Angaw Bogale, ${ }^{1}$ \\ Koku Sisay Tamirat, ${ }^{5}$ Mulat Birhanu Feleke, ${ }^{6}$ Muluken Azage, ${ }^{1}$ Dabere Nigatu, ${ }^{1}$ and Henry J. C. de Vries ${ }^{2}$ \\ ${ }^{1}$ College of Medicine and Health Sciences, Bahir Dar University, Bahir Dar, Ethiopia; ${ }^{2}$ Amsterdam UMC, University of Amsterdam, Department of \\ Dermatology, Amsterdam Institute for Infection and Immunity (AI\&I), Location Academic Medical Centre, Amsterdam, The Netherlands; \\ ${ }^{3}$ Department of Internal Medicine, College of Medicine and Health Sciences, Wollo University, Dessie, Ethiopia; ${ }^{4}$ Addis Alem Hospital, Bahir Dar, \\ Ethiopia; ${ }^{5}$ Department of Epidemiology and Biostatistics, Institute of Public Health, College of Medicine and Health Sciences, University of \\ Gondar, Gondar, Ethiopia; ${ }^{6}$ WagHimra Zone Health Department, Sekota, Ethiopia
}

\begin{abstract}
Countries like Ethiopia have had to make difficult decisions to balance between the demands of the COVID-19 pandemic and maintaining the essential health service delivery. We assessed the effect of preventive COVID19 measures on essential healthcare services in selected health facilities of Ethiopia. In a comparative cross-sectional study, we analyzed and compared data from seven health facilities over two periods: the pre-COVID-19 period before the first reported COVID-19 case in the country and during the COVID-19 period. Data were summarized using descriptive statistics and the independent $t$ test. During the COVID-19 period the average number of monthly patient visits in the emergency department, pediatrics outpatient, and adult outpatient dropped by $27 \%, 30 \%$, and $27 \%$, respectively compared with the pre-COVID-19 period. Family planning; institutional delivery; childhood immunization; antenatal care-, hypertension- and diabetic patient follow-up, did not vary significantly between pre-COVID-19 and during COVID-19. Moreover, the monthly average number of tuberculosis (TB) and HIV patients who visited health facilities for drug refill and clinical evaluation did not vary significantly during the two periods. In conclusion, the study highlights that the effect of public restrictions to mitigate the COVID-19 pandemic on essential care systems should be considered.
\end{abstract}

\section{INTRODUCTION}

Sever acute respiratory syndrome coronavirus 2 (SARSCoV-2) that causes coronavirus disease 2019 (COVID-19) has spread rapidly since the end of 2019 , to become a pandemic and a public health emergency of international concern. ${ }^{1,2}$ Countries around the world maximized their efforts to prevent the transmission of the virus and provide care for COVID-19 patients. However, the main challenge was to meet the demand imposed by the increasing number of COVID-19 patients, while maintaining the delivery of essential health services. ${ }^{3,4}$ A well-organized and prepared health system has the capacity to maintain equitable access to essential service delivery throughout an emergency period, limiting direct and indirect morbidity and mortality. ${ }^{4}$ Maintaining community trust in the essential health system capacity and safety in a time of emergency is key to ensure appropriate healthcare-seeking behavior and adherence to public health advice. ${ }^{5}$

When health systems are overwhelmed, both direct mortality from the pandemic and indirect mortality from preventable and treatable conditions increase substantially. Analyses from the 2014-2015 Ebola outbreak suggest that more deaths caused by malaria, measles, tuberculosis (TB), HIV/AIDS and health system failures outweighed deaths from Ebola. ${ }^{6-8}$ In May 2020, WHO surveyed the impact of COVID-19 on health services for noncommunicable diseases in 155 countries. Although the survey confirmed that the impact is global, low-income countries in particular proved to be the most affected. ${ }^{9}$

*Address correspondence to Seid Getahun Abdela, Department of Internal Medicine, College of Medicine and Health Sciences, Wollo University, P.O. Box 1145, Dessie, Ethiopia. E-mail: seidgech014@gmail.com
Countries like Ethiopia will need to make difficult decisions to balance the demands from the COVID-19 pandemic, while simultaneously engaging in strategic planning and coordinated action to maintain essential health service delivery, mitigating the risk of system collapse. ${ }^{4}$

The WHO prepared a guideline on how to continue essential services during the COVID-19 pandemic. The guideline recommends continuing vaccinations, chronic diseases follow-up, maternal and child healthcare, by taking in to consideration the extent of the pandemic in each locality. In areas with a relatively limited number of COVID-19 cases, the health system may have the capacity to maintain routine service delivery in addition to managing COVID-19 cases. But in case of increased caseload, and/or compromization of the health workforce, strategic shifts are required to ensure that increasingly limited resources provide maximum benefit for a population. ${ }^{10,11}$

Ethiopia, a country with $1.5 \%$ of the global population $(N=117,557,128)$, reported the first COVID-19 case on March 13, 2020. As of May 23, 2021 Ethiopia had registered only $0.16 \%$ of the global number of COVID-19 cases and $0.12 \%$ of the global deaths from the disease. ${ }^{12}$ Following the $\mathrm{WHO}$ advice after confirming the first case, the Ethiopian federal ministry of health $(\mathrm{MOH})$ initiated different prevention strategies such as a partial and selective lockdown, the prohibition of mass gatherings such as mass praying, and the closure of schools. At the same time, the $\mathrm{MOH}$ ensured the continuation of essential services, such as reproductive, maternal, neonatal, child, and adolescent health services, and the management of major communicable and noncommunicable diseases, surgical care, and emergency and critical care. ${ }^{13}$ A study in Ethiopia to study the effect of COVID-19 on essential health services over 8 weeks (4 weeks before and 4 weeks after the implementation of preventive measures) revealed that the case flow for almost all the services has declined. ${ }^{14,15}$ However, the results may not 
be generalizable since the study was limited to one referral hospital and had a short duration. A PubMed search revealed some publications on the effect of COVID-19 on specific health services (cancer, mental health, radiology service, and noncommunicable diseases), ${ }^{16-18}$ but the effect of COVID-19 on essential healthcare was not assessed. Therefore, here we study the effect of preventive COVID-19 measures on essential healthcare services in selected primary and tertiary care settings of Amhara region, Ethiopia. Such information may guide policy decisions and balance the demands imposed by the COVID-19 pandemic, while maintaining essential health services. This will also help in mitigation of the risk of health system collapse.

\section{MATERIALS AND METHODS}

Study design. A comparative cross-sectional study to assess essential services uptake before and during the COVID-19 pandemic. We used monthly aggregated data from July 7, 2019 to July 6, 2020 (one Ethiopian fiscal year).

Study setting and period. Amhara, the second most populous Regional State in the nation, is one of the 10 administrative regions in Ethiopia with an estimated 30 million inhabitants. ${ }^{19}$ Based on the report of the $\mathrm{MOH}$, more than $90 \%$ of the population in the region has access to health facility. The three-tier healthcare system ranges from primary healthcare units to higher tertiary level specialized hospitals. Primary healthcare units (PHCU) comprise health posts, health centers, and primary hospitals, serving 4,000 to 100,000 inhabitants. Secondary healthcare comprises general hospitals covering a population of 1-1.5 million people, and the tertiary care system comprise referral and specialized hospitals covering a population of 3.5-5 million people. All three healthcare systems provide essential healthcare services. ${ }^{20}$ In the Amhara region, there are 98 hospitals and 825 health centers providing essential health services. In this study, we included data from four hospitals and three PHCUs (Table 1).

Study population. Medical records of the visitors of the abovementioned health facilities from July 7, 2019 to July 6, 2020.

Data collection and sources of data. Data were retrieved from Health Management Information Systems (HMIS). The HMIS data from two periods were analyzed and compared: the pre-COVID-19 period from July 7, 2019 to March 8, 2020 (before the first reported COVID-19 case in Ethiopia); and the during COVID-19 period from March 9, to July 6, 2020 (after the first reported case in Ethiopia). We collected the following data: name and tier of visited healthcare facility, number of patients visits to different essential health services (such as number of patients who visited adult and pediatric emergency; outpatient surgical, medical, and pediatrics departments; the number of newly diagnosed HIV cases; number of visit to antiretroviral therapy (ART) clinic), and maternal and child care indicators (number of deliveries stratified by route, vaccinations, if applicable).

Data management. Data at district level from health facility and regional registers were entered in Microsoft Excel by two independent persons and cross-checked by the principal investigator. Data were imported in SPSS (Version 20) (Chicago, Illinois) for data quality assessment and analysis. The data were presented using descriptive statistics: frequency and measures of central tendency. The Independent $t$ test was used to compare means before and during the COVID-19 periods.

\section{RESULTS}

Adult medical and surgical emergency department patient flow. The average number of monthly patients in the emergency department in the pre-COVID-19 period was 4,932. During COVID-19, it dropped by $27 \%$ to 3,603 patients, and the lowest number of patients was documented in April 2020 (2,889), yet there was no significant difference in the number of emergency visits between the pre-COVID-19 and during COVID-19 periods $(P=0.3)$ (Figure 1). The emergency visit at tertiary level hospitals significantly lower during COVID-19 $(P=0.009)$ whereas the primary healthcare level emergency visit didn't vary $(P=0.08)$.

Outpatient patient flow. Overall, 185,746 children visited the pediatrics outpatient department (OPD). The number of children who visited the OPD during the pre-COVID-19 was 16,602 . This was significantly higher than the number of children $(12,106)$ who visited the OPD during the COVID-19 period, $(P=0.005)$. The number of pediatric outpatients visit at primary healthcare facility significantly decreased during COVID-19 $(P=0.04)$. The tertiary level hospitals' pediatric outpatients visit didn't vary significantly $(P=0.3)$. Similarly, more than half a million $(583,859)$ adult patients visited the outpatient departments overall. The average monthly

TABLE 1

Characteristics of health facilities in Amhara region included in the study

\begin{tabular}{|c|c|c|}
\hline Name of health facility & Catchment area population & Number of health professionals \\
\hline Azezo health center & 69,628 & $\begin{aligned} \text { Nurses }=15, \text { Midwives } & =4, \text { Pharmacists }=13, \text { Health officer }=4, \text { and Laboratory } \\
& \text { technologists and technicians }=4\end{aligned}$ \\
\hline Adisalem Hospital & 300,000 & $\begin{array}{l}\text { General practitioners }=19, \text { Specialists }=4, \text { Nurses }=38, \text { Midwives }=16 \text {, } \\
\text { Pharmacists }=10 \text { and Laboratory technologists and technicians }=10\end{array}$ \\
\hline BahirDar health center & 76,343 & $\begin{array}{l}\text { General practitioner }=1 \text {, Nurses }=18, \text { health officers }=8, \text { Midwives }=7 \text {, } \\
\text { Pharmacy }=4 \text { and Laboratory technologists and technicians }=5\end{array}$ \\
\hline Haik health center & 71,829 & $\begin{array}{c}\text { Nurses }=13, \text { Midwives }=6, \text { Health officers, Pharmacists, Laboratory } \\
\text { technologists, and technicians }=5\end{array}$ \\
\hline Tefera Hailu General hospital & Above 1 million & $\begin{array}{c}\text { General practitioners }=17, \text { Specialists }=4 \text {, Nurses }=93, \text { Midwives }=24 \text {, and } \\
\text { Laboratory technologists and technicians }=12\end{array}$ \\
\hline $\begin{array}{l}\text { Dessie comprehensive } \\
\text { specialized hospital }\end{array}$ & Above 8 million & $\begin{array}{l}\text { General practitioners }=85, \text { Specialists }=42, \text { Nurses }=210, \text { Midwives }=47, \\
\text { Pharmacists }=48, \text { and Laboratory technologists and technicians }=38\end{array}$ \\
\hline Tibebe Ghion Teaching Hospital & Above 5 million & $\begin{array}{l}\text { General practitioners }=16, \text { Specialists }=123, \text { Nurses }=284, \text { Midwives }=69, \\
\text { Pharmacists }=42, \text { and Laboratory technologists and technicians }=48\end{array}$ \\
\hline
\end{tabular}

These health facilities were selected after considering their location and convenience. 


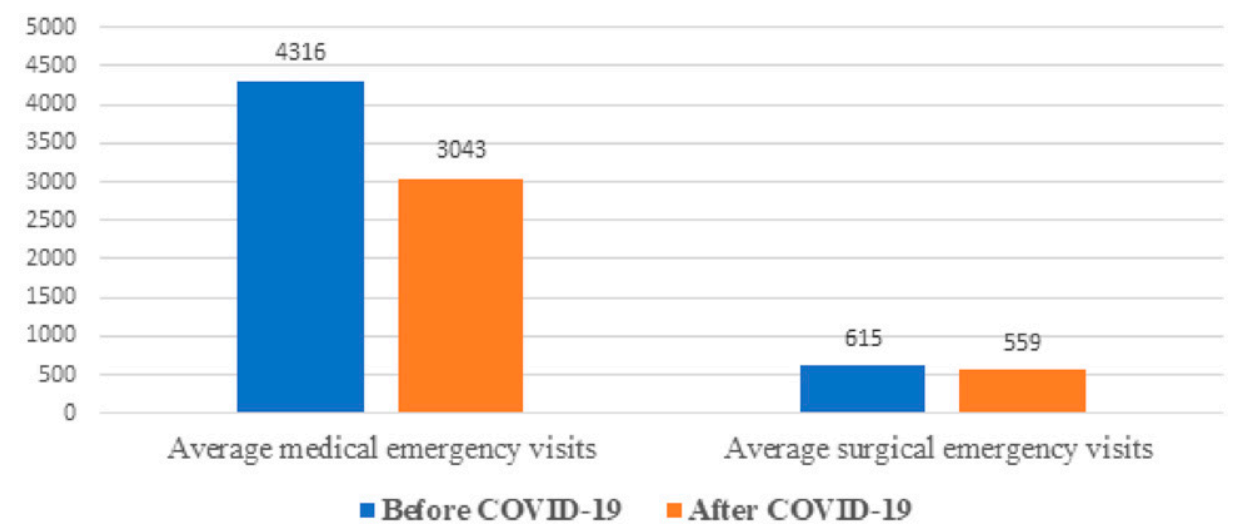

FIGURE 1. Monthly medical and surgical emergency visits pre-COVID-19, and during the COVID-19 pandemic from selected health facilities in Amhara region, Ethiopia, from July 7, 2019 to March 8, 2020, and from March 9, 2020 to July 6, 2020. This figure appears in color at www.ajtmh.org.

number of adults who visited the OPD during the preCOVID-19 period was 52,165 . This number was significantly higher than the number of adults $(38,120)$ who visited the OPD during the COVID-19 period (Figure 2) $(P=0.014)$. The number of adult OPD visit at primary healthcare facility didn't vary significantly pre-COVID-19 and during COVID-19 period $(P=0.2)$. The tertiary level hospitals adult OPD visit during the COVID-19 period was significantly lower than the preCOVID-19 period $(P=0.02)$.

Maternal, child health, and family planning visits. In the pre COVID-19 periods, the monthly average visits for shortterm family planning was 1,530 and dropped by $14 \%$ to 1,328 during the COIVD-19 period. Yet, the monthly average longterm family planning utilization was 256 and 259, respectively (Figure 3). Both, short and long-term family planning did not vary significantly between pre-COVID-19 and during COVID-19.

The mean monthly institutional delivery was 1,133 before the pandemic and in the pandemic months it has increased by $7.5 \%$ to 1,226 (Figure 4). The institutional delivery did not vary significantly between pre COVID-19 and during COVID19.The average institutional delivery at primary healthcare facilities significantly increased during the COVID-19 period compared with pre-COVID-19 period $(P=0.03)$.

Antenatal care follow-up. The monthly mean of antenatal care (ANC) follow-up in pre-COVID-19 months was 910 and the pandemic period 941 . The lowest number of first
ANC visitors to health facility was seen in April (572) with about $38 \%$ decrease from the fiscal year mean with a rebound in May and June by 114 and 128\% respectively (Figure 5). The ANC visit did not vary significantly between pre COVID-19 and during COVID-19.

Vaccination program. The mean monthly number of children that received full immunization pre-COVID-19 and during the pandemic period was 468 and 469 , respectively.

Chronic disease services uptake. In the pre-COVID-19 period, the monthly average diabetic patient visit was 1,078 and during the pandemic it dropped significantly by $31 \%$ to $745(P=0.001)$. Yet, the monthly average hypertension patients visiting health facilities dropped with $12 \%$ not significantly $(P=0.2)$ from 828 to 728 , respectively.

HIV care and treatment and TB case detection. The mean number of HIV/AIDS patients who visited health facilities for drug refill and clinical evaluation on a monthly basis during the pre-COVID-19 period was 720 with no significant difference during the COVID-19 period (726). Yet, the mean number of newly diagnosed HIV/AIDS patients dropped by $43 \%$ during the COVID-19 period time compared with the pre-COVID-19 period.

The monthly average new TB case detection rate in the health centers and primary hospital was 93 before the lockdown and during the pandemic time, it decreased by only $4 \%$ to 89 .

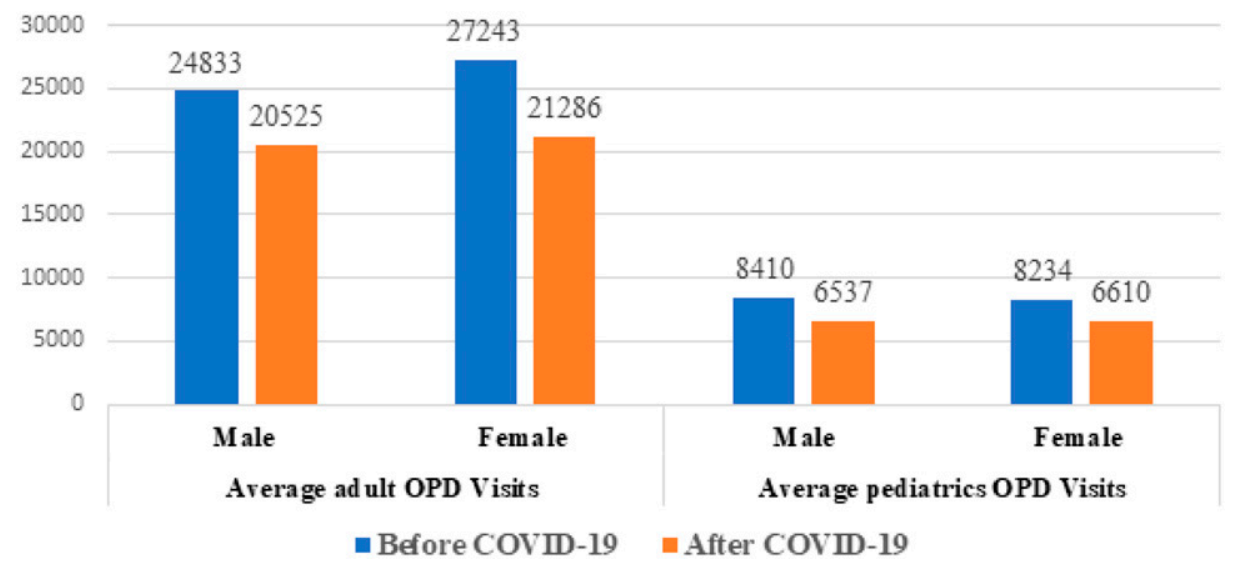

FIGURE 2. Monthly adult and pediatric outpatient department visitors pre-COVID-19, and during the COVID-19 pandemic from selected health facilities in Amhara region, Ethiopia, from July 7, 2019 to March 8, 2020, and from March 9, 2020 to July 6, 2020. This figure appears in color at www.ajtmh.org. 
Pattterns of average monthly short and long term family planning service utilization

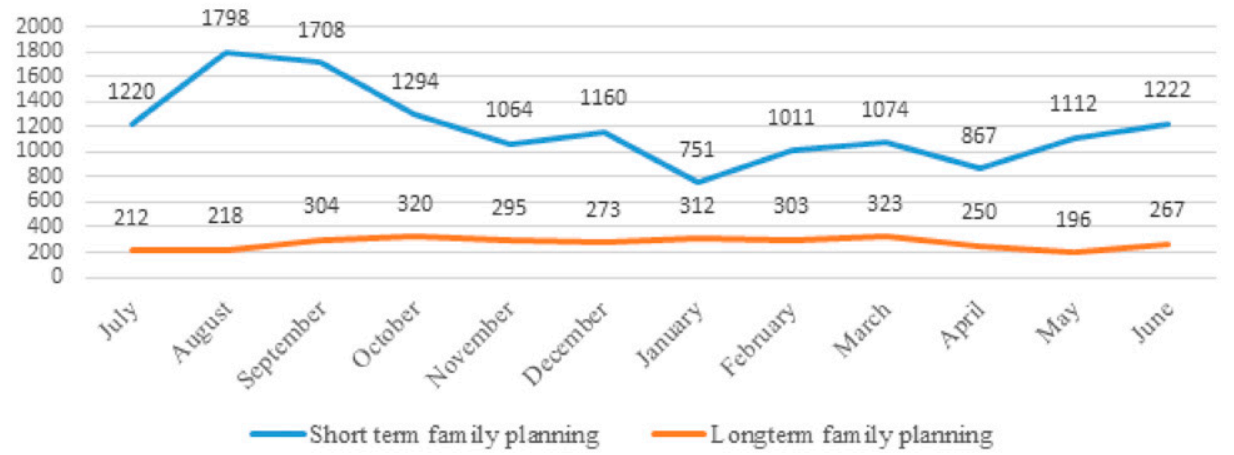

FIGURE 3. Short- and long-term family planning uptakes in pre-COVID-19, and during the COVID-19 pandemic from selected health facilities in Amhara region, Ethiopia, from July 7, 2019 to March 8, 2020, and from March 9, 2020 to July 6, 2020. This figure appears in color at www.ajtmh.org.

\section{DISCUSSION}

Here we quantified the effect of the COVID-19 pandemic on the essential health services and the recovery of some health services. Patient flow for most essential health services (adult and pediatric outpatient visits, childhood immunization, surgical and medical emergency, chronic noncommunicable diseases) declined after the first COVID-19 case notification mid-March in Ethiopia. The lowest patients flow was documented in the subsequent month, April 2020. After this first decline, there was rapid recovery in patient flow for almost all essential health services (neonatal and child care, maternal health, chronic noncommunicable diseases, TB, and HIV).

Evidence from the 2013 to 2016 Ebola virus outbreak in western African countries indicated the negative, indirect effects of the epidemic on essential health services. ${ }^{10,21}$ Our study confirms an initial decline in the access of essential health services in the beginning of the COVID-19 pandemic (April 2020). The initial rigorous preventive measures including restriction of public transport and the public fear of contracting the disease may have contributed to the decreased client/patient flow. This may have directly or indirectly increased mortality and morbidities from treatable and preventable conditions. Yet, the disruption did not last long and in months of May and June the patient flow rebounded back to the prepandemic level. A study by Abdela et al., in one hospital in Ethiopia on essential healthcare visits, 4 weeks before and 4 weeks after the implementation of preventive measures concluded the decline of all essential healthcare services during COVID-19 preventive measure. ${ }^{14}$ The main limitation of the study was the short observation period that only included the first month of the pandemic coinciding with the most strict lockdown period so far.

The CDC and other reports have documented a marked decrease in vaccinations and primary prevention services during the pandemic. ${ }^{21-23}$ Here we found that the average childhood immunization in April decreased by $11 \%$ from the annual averages, 4 months later, the average childhood immunization was higher than the prepandemic period by about $11 \%$. Also, there is no difference in the monthly average full immunizations before the pandemic (468) and after the pandemic (469), which is reassuring for the continuity of the health system.

In April 2020, Riley et al., used a mathematical model to forecast that "a $10 \%$ proportional decline in use of short- and long-acting contraceptive methods in low- and middleincome countries (LMICs) due to reduced access to healthcare services may lead to an additional 49 million women with an unmet need for modern contraceptives and an additional 15 million unintended pregnancies over the course of a year." They also predicted that around a 10\% decrease in maternal and new born care could result in catastrophic implications on the health of mothers and new born. ${ }^{24}$ In our

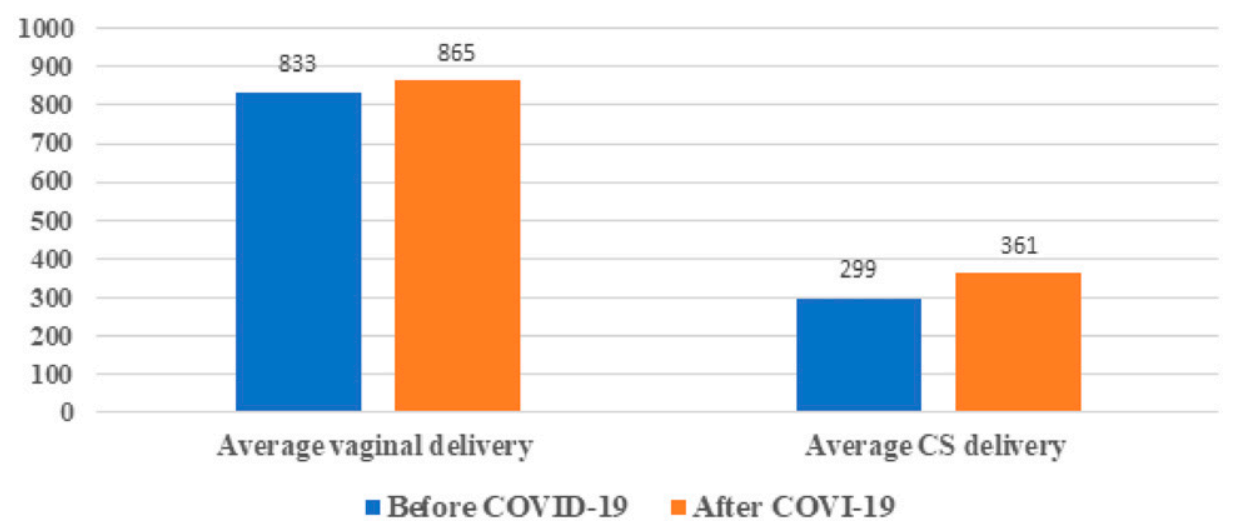

FIGURE 4. Average monthly vaginal and CS delivery pre-COVID-19, and during the COVID-19 pandemic from selected health facilities in Amhara region, Ethiopia, from July 7, 2019 to March 8, 2020, and from March 9, 2020 to July 6, 2020. This figure appears in color at www.ajtmh.org. 


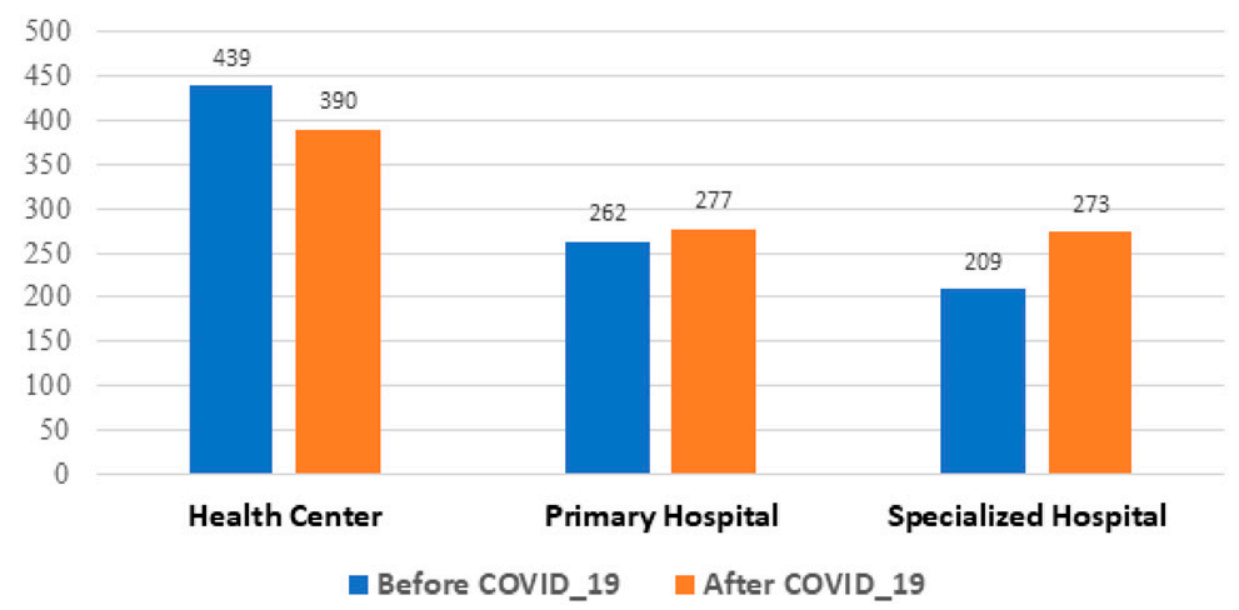

FIGURE 5. Average Antenatal care follow-up during pre-COVID-19, and during the COVID-19 pandemic period from selected health facilities in Amhara region, Ethiopia, from July 7, 2019 to March 9, 2020, and from March 13, 2020 to July 6, 2020. This figure appears in color at www.ajtmh.org.

observation, the pandemic did not show major effect on the number of institutional delivery and family planning services.

In June 2020, the WHO report on "rapid assessment of services delivery for non-communicable disease" stated that in countries where community transmission of COVID-19 is confirmed there is $66 \%$ and $64 \%$ of disruption services for hypertension and diabetes management, respectively. ${ }^{25}$ In our study on the monthly follow-up visit, there was only $18 \%$ and $31 \%$ declines in both hypertension and diabetes services, respectively.

There were concerns that countries with high burdens of TB and HIV/AIDS might be have difficulties in maintaining continuity of (quality) care to their patients, because of lockdowns restricting clinic access and the redeployment of financial and human resources away from HIV/AIDS and TB. ${ }^{26,27}$ Mohammed et al., reported that the new case detection of TB from one of the regions in Ethiopia from April to June (during the pandemic) has decreased about three times from the previous reporting periods. The number of new TB detection and HIV clinic follow-up was not affected by the pandemic in our study. However, the number of new HIV diagnosis decreased significantly during the pandemic period.

The $\mathrm{MOH}$ observed the worrisome declines in health services early in April. In collaboration with the regional health bureaus, the $\mathrm{MOH}$ circulated a directive for sustaining essential health service during the pandemic. ${ }^{27}$ In addition, in April 24, the $\mathrm{MOH}$ after giving a press release on the importance of maintaining essential health services, assigned a team of experts and decision makers to monitor the continuum of essential health services. ${ }^{28}$ We think that the reasons for the sustained and recovery of the essential health service are two factors. Firstly, the swift response by the $\mathrm{MOH}$ and regional health bureaus and secondly, the slow progression of the pandemic which never overwhelmed the health system. ${ }^{12}$ Apart from the essential health system, the authors recommend a further study to explore the full

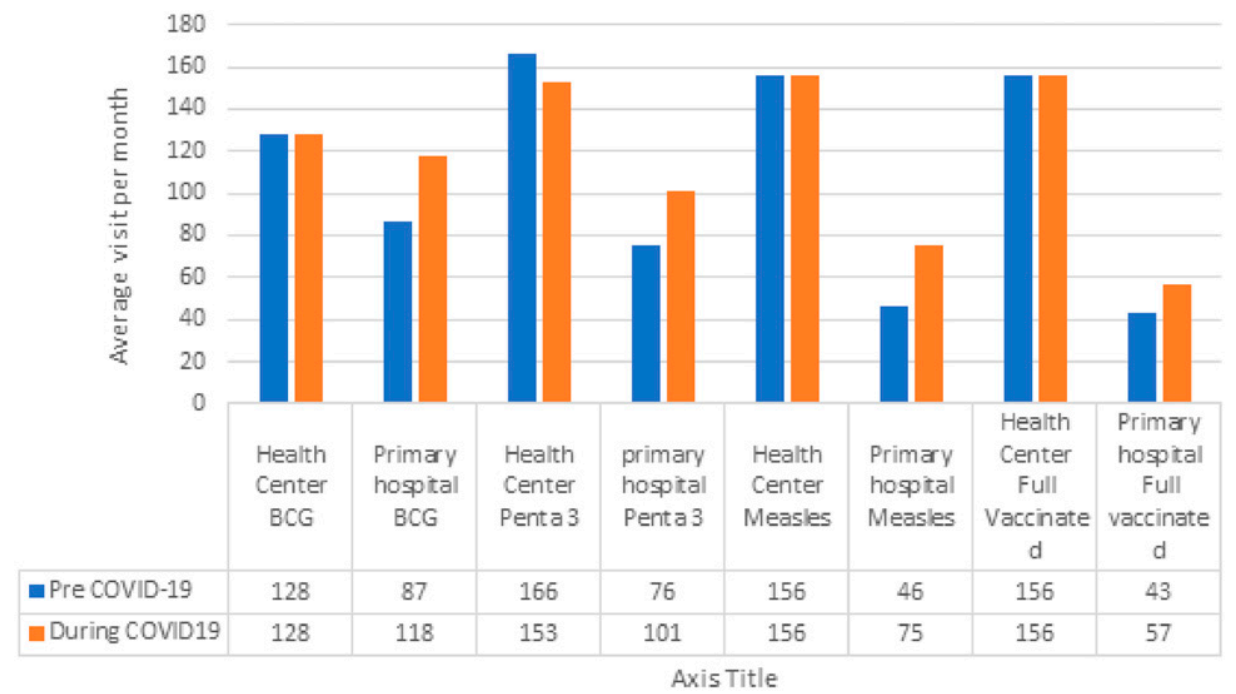

FIGURE 6. Average monthly visits for vaccinations during pre-COVID-19, and during the COVID-19 pandemic period from selected health facilities in Amhara region, Ethiopia, from July 7, 2019 to March 8, 2020, and from March 9, 2020 to July 6, 2020. This figure appears in color at www.ajtmh.org. 
Patterns of Tuberculosis case detection in health centers and primary hospitals

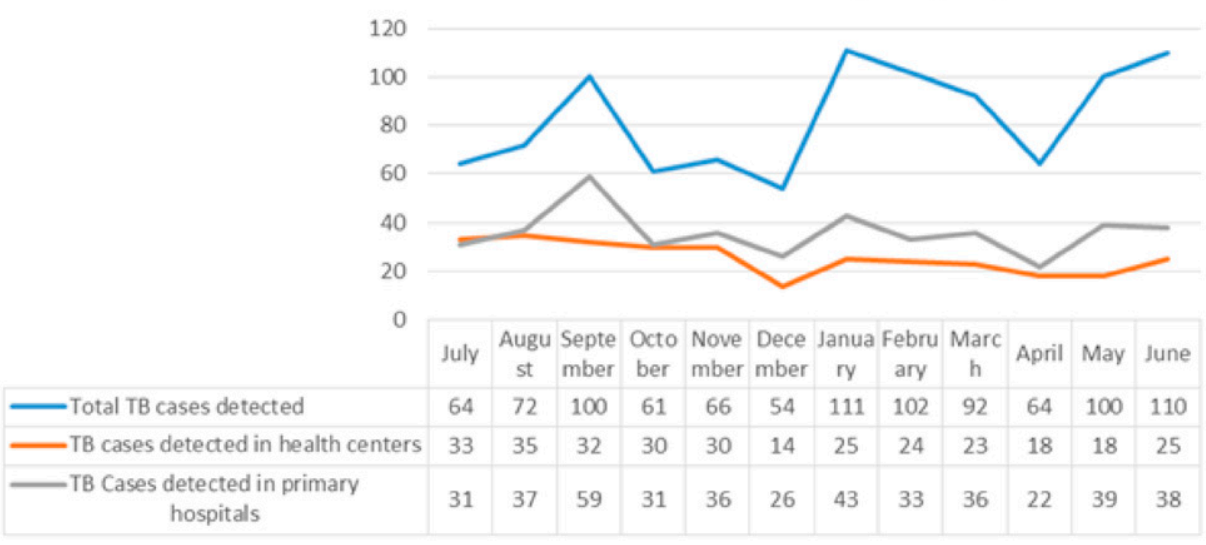

FIGURE 7. Patterns of new TB case detection during pre-COVID-19, and during the COVID-19 pandemic period from selected health facilities in Amhara region, Ethiopia, from July 7, 2019 to March 8, 2020, and from March 9, 2020 to July 6, 2020. This figure appears in color at www.ajtmh.org.

effect of the pandemic in the disease prevention programs, including the health extension program.

A strength of the study is its multicenter design involving different tiers of health facilities, therefore, it is representative of the Ethiopian healthcare units. Second, the study has looked at the data for an extensive whole fiscal year documenting the service of 8 months pre-COVID-19 period and 4 months during COVID-19 period. And third, we adhered to the STROBE guidelines (Strengthening the Reporting of Observational Studies in Epidemiology). ${ }^{29}$

The limitation of the study is that the study population did not include frontline health workers (health post) which are responsible for most of the preventive health services (family planning and immunization). Ideally, the pandemic timeframe of the periods (esp. during COVID-19 period) should be extended, to assess the effects later on during the COVID19 pandemic. Additionally, comparison with the same months of previous years would account for the seasonality of some of the primary healthcare demands. But the 1-year data has also shown there is no significant variation in annual average patient flow, which is reassuring for the influence of seasonality.

Given that the pandemic is ongoing, such findings are of high interest to clinicians and policy makers in their effort to improve the resilience of health systems and hospitals during pandemics or similar disruptive events, including extreme climatic events. In conclusion, the study highlights the need for a strong program to assure essential healthcare components, while preventive procedures to mitigate a pandemic are imposed. The effect of public restrictions on essential care systems should be considered.

Received March 28, 2021. Accepted for publication August 12, 2021.

Published online September 20, 2021.

Acknowledgments: We thank all health facility administrations and staffs of the archive/card room for providing us the access and their role in data extraction. The American Society of Tropical Medicine and Hygiene has waived the Open Access fee for this article due to the ongoing COVID-19 pandemic and has assisted with publication expenses.
Authors' addresses: Wendemagegn Enbiale, College of Medicine and Health Sciences, Bahir Dar University, Bahir Dar, Ethiopia, and Amsterdam UMC, University of Amsterdam, Department of Dermatology, Amsterdam Institute for Infection and Immunity (AI\&I), Location Academic Medical Centre, Amsterdam, The Netherlands, E-mail: wendemagegnenbiale@gmail.com. Seid Getahun Abdela, Department of Internal Medicine, College of Medicine and Health Sciences, Wollo University, Dessie, Ethiopia, E-mail: seidgech014@ gmail.com. Meaza Seyum, Addis Alem Hospital, Bahir Dar, Ethiopia, E-mail: meazasm@gmail.com. Dereje Bedanie, Kasawmar Angaw Bogale, Muluken Azage, and Dabere Nigatu, College of Medicine and Health Sciences, Bahir Dar University, Bahir Dar, Ethiopia, E-mails: derejebedane@gmail.com, kassawmarangaw@gmail.com, akiyamuluken19@gmail.com, and dabenigatu@gmail.com. Koku Sisay Tamirat, Department of Epidemiology and Biostatistics, Institute of Public Health, College of Medicine and Health Sciences, University of Gondar, Gondar, Ethiopia, E-mail: kokusisay23@gmail. com. Mulat Birhanu Feleke, WagHimra Zone Health Department, Sekota, Ethiopia, E-mail: bmmulat@yahoo.com. Henry J. C. de Vries, Amsterdam UMC, University of Amsterdam, Department of Dermatology, Amsterdam Institute for Infection and Immunity (AI\&I), Location Academic Medical Centre, Amsterdam, The Netherlands, E-mail: h.j.devries@amsterdamumc.nl.

This is an open-access article distributed under the terms of the Creative Commons Attribution (CC-BY) License, which permits unrestricted use, distribution, and reproduction in any medium, provided the original author and source are credited.

\section{REFERENCES}

1. Castle S et al., 2020. Coronavirus Live Updates: W.H.O. Declares Pandemic as Number of Infected Countries Grows. The-New-York-Times, March 11, 2020. Available at: https:// www.nytimes.com/2020/03/11/world/coronavirus-news. html\#link-682e5b06.

2. WHO, 2020. Statement on the Second Meeting of the International Health Regulations (2005) Emergency Committee Regarding the Outbreak of Novel Coronavirus (2019$n$ CoV). Geneva, Switzerland: World Health Organization. Available at: https://www.who.int/news-room/detail/3001-2020-statement-on-the-second-meeting-of-the-intern ational-health-regulations-(2005)-emergency-committeeregarding-the-outbreak-of-novel-coronavirus-(2019-n cov). Accessed March 30, 2020.

3. Kieny MP, Evans DB, Schmets G, Kadandale S, 2014. Healthsystem resilience: reflections on the Ebola crisis in western Africa. Bull World Health Organ 92: 850. 
4. WHO, 2020. COVID-19: Operational Guidance for Maintaining Essential Health Services during an Outbreak. Geneva, Switzerland: World Health Organization.

5. Elston JWT et al., 2015. Impact of the Ebola outbreak on health systems and population health in Sierra Leone. J Public Health (Bangkok) 38: 673-678.

6. Elston JWT, Cartwright C, Ndumbi P, Wright J, 2017. The health impact of the 2014-15 Ebola outbreak. Public Health 143: 60-70.

7. Parpia AS, Ndeffo-Mbah ML, Wenzel NS, Galvani AP, 2016. Effects of response to 2014-2015 Ebola outbreak on deaths from malaria, HIV/AIDS, and tuberculosis, West Africa. Emerg Infect Dis 22: 433.

8. Delamou A et al., 2017. Effect of Ebola virus disease on maternal and child health services in Guinea: a retrospective observational cohort study. Lancet Glob Health 5: e448-e457.

9. WHO, 2020. Rapid Assessment of Service Delivery for NCDs During the COVID-19 Pandemic. Geneva, Switzerland: World Health Organization. Available at: https://www.who.int/publications $/ \mathrm{m} /$ item/rapid-assessment-of-service-delivery-for-ncds-during-thecovid-19-pandemic. Accessed November 13, 2020.

10. Sochas L, Channon AA, Nam S, 2017. Counting indirect crisisrelated deaths in the context of a low-resilience health system: the case of maternal and neonatal health during the Ebola epidemic in Sierra Leone. Health Policy Plan 32 (Suppl_3): iii32-iii9.

11. WHO, 2020. Operational Guidance for Maintaining Essential Health Services during an Outbreak. Geneva, Switzerland: World Health Organization. Available at: https://www.who.int/publicationsdetail/covid-19-operationalguidance-for-maintai ning-essentialhealth-services-during-anoutbreak. Accessed April 26, 2020.

12. Worldometer, 2021. Coronavirus Cases. Available at: https:// www.worldometers.info/coronavirus/. Accessed March 14, 2021.

13. Ethiopia $\mathrm{MOH}, 2019$. Essential Health Service Package of Ethiopia. November 3, 2019, Addis Ababa Ethiopia. Available at: https://www.humanitarianresponse.info/en/operations/ethiopi a/document/essential-health-services-package-ethiopia-2019. Accessed February 20, 2021.

14. Abdela SG, Berhanu AB, Ferede LM, van Griensven J, 2020. Essential healthcare services in the face of COVID-19 prevention: experiences from a referral hospital in Ethiopia. Am J Trop Med Hyg 103: 1198-1200.

15. Palmer K, Monaco A, Kivipelto M, Onder G, Maggi S, Michel JP, Prieto R, Sykara G, Donde S 2020. The potential long-term impact of the COVID-19 outbreak on patients with noncommunicable diseases in Europe: consequences for healthy ageing. Aging Clin Exp Res 32: 1189-1194

16. Chen $S$ et al., 2020. The early impact of COVID-19 on mental health and community physical health services and their patients' mortality in Cambridgeshire and Peterborough, UK. J Psychiatr Res 131: 244-254.
17. Dinmohamed AG et al., 2020. The impact of the temporary suspension of national cancer screening programmes due to the COVID-19 epidemic on the diagnosis of breast and colorectal cancer in the Netherlands. J Hematol Oncol 13: 147.

18. Ethiopian Central Statistical Agency, 2013. Population Projections for Ethiopia 2007-2037. Addis Ababa. Available at: https://www.statsethiopia.gov.et/wp-content/uploads/2019/ 05/ICPS-Population-Projection-2007-2037-produced-in-2012. pdf. Accessed May 3, 2019.

19. FMOH, 2010. Health Sector Development Program IV (2010/ 2011-2014/2015). Addis Ababa, Ethiopia: Federal Ministry of Health of Ethiopia.

20. Hall KS et al., 2020. Centring sexual and reproductive health and justice in the global COVID-19 response. Lancet 395: 1175-1177.

21. Bietsch K, Williamson J, Reeves M, 2020. Family planning during and after the West African Ebola crisis. Stud Fam Plann 51: 71-86.

22. CDC, 2020. Effects of the COVID-19 Pandemic on Routine Pediatric Vaccine Ordering and Administration - United States, 2020. Available at: https://www.cdc.gov/mmwr/volumes/69/ $\mathrm{wr} / \mathrm{mm6919e2.htm.} \mathrm{Accessed} \mathrm{May} \mathrm{4,2020.}$

23. Samai O, Sengeh $P, 1997$. Facilitating emergency obstetric care through transportation and communication, Bo, Sierra Leone. Bo PMM Team. Int J Gynaecol Obstet 59: S157-S164.

24. Riley T, Sully E, Ahmed Z, Biddlecom A, 2020. Estimates of the potential impact of the COVID-19 pandemic on sexual and reproductive health in low- and middle-income countries. Int Perspect Sex Reprod Health 46: 73-76.

25. World Health Organization, 2020. Final Results Rapid Assessment of Service Delivery for Non Communicable Diseases (NCD) During the Covid-19 Pandemic. WHO NCD Department. Available at: https://www.who.int/publications/m/item/rapidassessment-of-service-delivery-for-ncds-during-the-covid-19pandemic. Accessed January 4, 2021

26. Pang Y, Liu Y, Du J, Gao J, Li L, 2020. Impact of COVID-19 on tuberculosis control in China. Int $J$ Tuberc Lung Dis 24: 545-547.

27. Mohammed H, Oljira L, Roba KT, Yimer G, Fekadu A, Manyazewal T, 2020. Containment of COVID-19 in Ethiopia and implications for tuberculosis care and research. Infect Dis Poverty 9: 131.

28. $\mathrm{MOH}, 2020$. It was Stated that Regular Health Services Should be Strengthened Where They Were. Addis Ababa, Ethiopia: Ministry of Health-Ethiopia. Available at: http:// www.moh.gov.et/ejcc/am/node/205. Accessed February 6, 2011.

29. Poorolajal J, Cheraghi Z, Irani AD, Rezaeian S, 2011. Quality of cohort studies reporting post the strengthening the reporting of observational studies in epidemiology (STROBE) statement. Epidemiol Health 33: e2011005. 